SLAC-PUB-8138

March, 1999

\title{
Simulation Studies of Main Linac Steering in the Next Linear Collider ${ }^{1}$

\author{
P. TENEnBAuM
} \\ Stanford Linear Accelerator Center, Stanford University, Stanford, CA
}

\begin{abstract}
In order to achieve the design luminosity of the Next Linear Collider, the main linac must accelerate trains of bunches from $10 \mathrm{GeV}$ to $500 \mathrm{GeV}$ while preserving vertical normalized emittances on the order of $0.05 \mathrm{~mm} . \mathrm{mrad}$. We describe a set of simulation studies, performed using the program LIAR, comparing several algorithms for steering the main linac; the algorithms are compared on the basis of emittance preservation, convergence speed, and sensitivity to BNS phase profile. The effects of an ATL mechanism during the steering procedure are also studied.
\end{abstract}

Contributed to 1999 IEEE Particle Accelerator Conference, New York, New York, March 29 - April 2, 1999

\footnotetext{
${ }^{1}$ Work supported by the Department of Energy, Contract DE-AC03-76SF00515.
} 


\title{
SIMULATION STUDIES OF MAIN LINAC STEERING IN THE NEXT LINEAR COLLIDER ${ }^{\dagger}$
}

\author{
P. Tenenbaum ${ }^{\ddagger}$ \\ Stanford Linear Accelerator Center, Stanford University, Stanford, CA \\ - Each structure contains a beam position monitor at \\ each end with a resolution of 5 microns in $x$ and $y$ \\ for a single bunch with a charge of $10^{10}$.
}

Abstract

In order to achieve the design luminosity of the Next Linear Collider, the main linac must accelerate trains of bunches from $10 \mathrm{GeV}$ to $500 \mathrm{GeV}$ while preserving vertical normalized emittances on the order of $0.05 \mathrm{~mm}$.mrad. We describe a set of simulation studies, performed using the program LIAR, comparing several algorithms for steering the main linac; the algorithms are compared on the basis of emittance preservation, convergence speed, and sensitivity to BNS phase profile. The effects of an ATL mechanism during the steering procedure are also studied.

\section{INTRODUCTION}

The Next Linear Collider (NLC) is a single-pass electronpositron collider capable of achieving a luminosity of $10^{34} \mathrm{~cm}^{-2} \mathrm{sec}^{-1}$ at a center-of-mass energy of $1 \mathrm{TeV}[1]$. The NLC uses a pair of X-Band ( $f=11.424 \mathrm{GHz})$ linear accelerators, with approximately 5,000 RF structures and 750 quadrupoles in each linac, to accelerate the beams from 10 to $500 \mathrm{GeV}$. The total length of each linac is over 10 kilometers.

In order to achieve the desired luminosity, each linac must accelerate a 270 nanosecond train of 95 bunches on each $120 \mathrm{~Hz}$ machine cycle, and must preserve an incoming normalized emittance which can be as small as 0.03 mm.mrad. Novel structure designs can mitigate the emittance dilution due to long-range wakefields [2]; this leaves dispersion and short-range wakefields from the structures as the primary causes of emittance dilution. In both wakefields and dispersion, the emittance dilution can be controlled through proper alignment of the accelerator. The NLC design calls for an unprecedented emphasis on measurement and correction of misalignments:

- Each quad is supported on a remote-controlled translation stage capable of $\pm 2 \mathrm{~mm}$ motions in $x$ and $y$, with submicron step sizes

- Each RF structure girder (3 structures) is supported on a remote-controlled translation stage capable of \pm 2 mm motions in $x$ and $y$ at each end of each girder, with micron step sizes

- Each quad contains a beam position monitor with a resolution of 1 micron in $x$ and $y$ for a single bunch with a charge of $10^{10}$

\footnotetext{
$\dagger$ Work supported by the Department of Energy, contract DE-AC03$76 \mathrm{SF} 00515$

‡Email:quarkpt@slac.stanford.edu
}

We consider three algorithms for converting beam position information in the quad and structure BPMs into changes in translation stage positions.

\section{DESCRIPTION OF THE ALGORITHMS}

\section{1 "Canonical" Algorithm}

The algorithm used to study beam-based alignment in the 1996 NLC study divides the linac into $N$ segments containing equal numbers of quads (in practice, 14 segments with approximately 50 quads per segment) and uses the quadrupole BPMs to compute a set of magnet moves which minimizes (in a least-square sense) the RMS BPM orbit. In order to prevent the magnet movers "ranging out," the algorithm simultaneously seeks to minimize the RMS magnet motion, resulting in an overconstrained fit. Once the quads have been moved, each structure girder in the segment is then moved to zero the average of the 6 structure BPMs on the girder.

In this algorithm the least-squares engine uses the wakefree optics model to predict the response to quad moves, and assumes that girder moves only change the readings of BPMs on the girder. Because the wakefield contribution is not included in the calculation, it is necessary in real life to iterate the algorithm on each segment several times before moving on to the next segment, and to pick segments which are short relative to the characteristic growth distance of wakefield instabilities.

In order to match the alignment from one segment into another, the magnets at the endpoints of a segment are held fixed in position: a steering magnet at the first quad is used to steer the beam into the last quad, and its value is determined as part of the least-squares fit. Thus the algorithm results in a piecewise-straight alignment, with kinks at the endpoints of segments.

\section{2 "Canonical" Algorithm with MICADO}

Under some circumstances the "Canonical" algorithm will leave an RMS orbit which is larger than the BPM resolution. Errors in positioning of the many quads will sometimes conspire to produce a betatron component to the orbit. In order to further reduce this, the "Canonical" algorithm can be followed by a MICADO algorithm [3], which attempts to identify the minimum number of magnet moves 
which produce the greatest improvement in the orbit. For the purposes of this simulation, the MICADO algorithm was constrained to use no more than 7 magnets, and to seek an RMS orbit tolerance of 1 micron. In execution several iterations of the "canonical" algorithm would be performed on an alignment segment, followed by several MICADO algorithms.

\section{3 "French Curve” Algorithm}

The "canonical" algorithm inconveniently requires corrector magnets at the endpoints of each segment. An algorithm was sought which would not require such magnets, but still permitted the segment-to-segment alignment matching provided by the correctors. The "French Curve" algorithm is very similar to the "canonical" algorithm; however, no correctors are used, and instead after a segment is aligned the next segment is selected starting in the middle of the most recent one. Thus the alignment is performed on full segments but advances down the linac in half-segments, resulting in a smooth alignment without correctors.

\section{SIMULATION STUDIES}

Each of the 3 algorithms was studied with LIAR [4], a linear accelerator simulation program which performs tracking with transverse and longitudinal wakefields from RF structures. The general conditions of the simulation are described in the section above and in Table 1.

Table 1: General parameters used in the simulations.

\begin{tabular}{|c|c|}
\hline Parameter & Value \\
\hline \hline Bunch \\
Charge & $1.1 \times 10^{10}$ \\
\hline $\begin{array}{c}\text { Quad-BPM } \\
\text { Offset }\end{array}$ & 2 microns \\
\hline $\begin{array}{c}\text { Struct.-BPM } \\
\text { Offset }\end{array}$ & 0 microns \\
\hline $\begin{array}{c}\text { Incoming } \\
\gamma \epsilon_{y}\end{array}$ & $0.04 \mathrm{~mm} . \mathrm{mrad}$ \\
\hline
\end{tabular}

\subsection{Mover Step Size}

Figure 1 shows the emittance dilution of each algorithm as a function of the magnet mover step size. In each case the algorithm was permitted to iterate to convergence (see next section). While MICADO can improve the performance of the "canonical" algorithm at large step sizes, it cannot reduce the residual emittance growth which occurs for small step sizes. The "french curve" algorithm has a smaller emittance growth for perfect movers than the "canonical;" its emittance dilution is also a weaker function of mover step size.

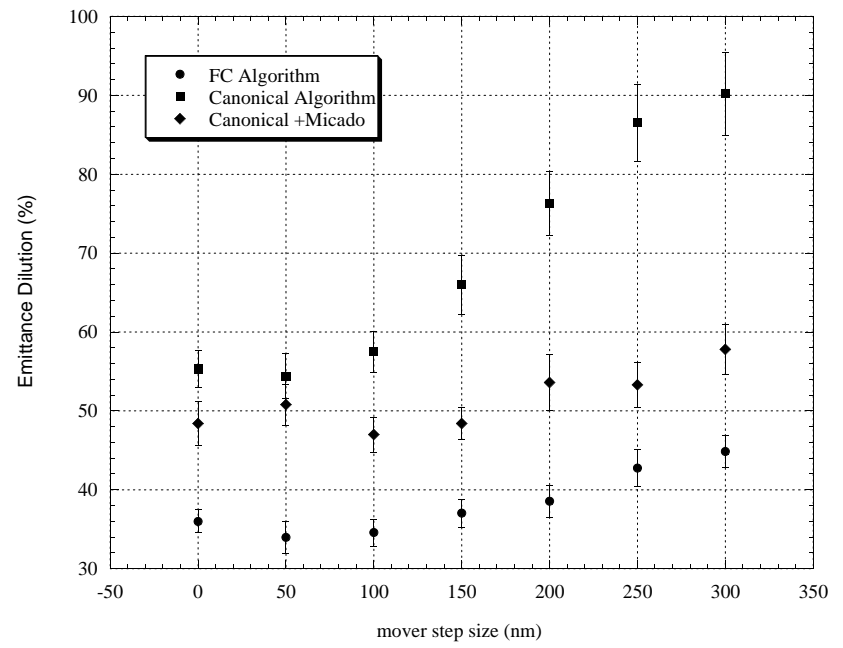

Figure 1: Emittance dilution as a function of magnet mover step size for 3 main linac steering algorithms.

\subsection{Convergence Speed}

Figure 2 shows the number of iterations required to reach convergence for "canonical" and "french curve" algorithms. While the latter algorithm required fewer iterations per segment, it also requires twice as many segments as the "canonical" algorithm, and is thus somewhat slower in terms of time.

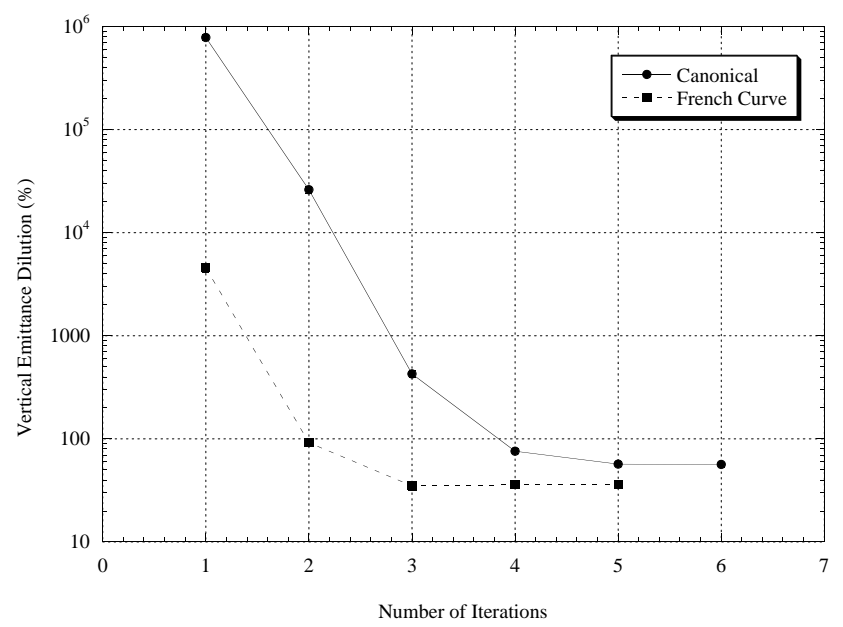

Figure 2: Emittance dilution as a function of number of iterations per segment for "canonical" and "french curve" algorithms.

\subsection{Energy Overhead}

In order to reduce the impact of incoming beam jitter on emittance, the NLC linacs will be operated with a substantial head-tail energy difference [5], which is parameterized here as linac energy overhead (linac voltage in excess of that needed to achieve the desired energy at extraction). Figure 3 shows that the emittance dilution increases linearly for both "canonical" and "french curve" algorithms 
with energy overhead (note that this is contrary to the jitter behavior: more energy overhead results in less emittance dilution for a bunch executing a betatron oscillation down the full length of the linac). However the "french curve" performance is better for all values of energy overhead considered.

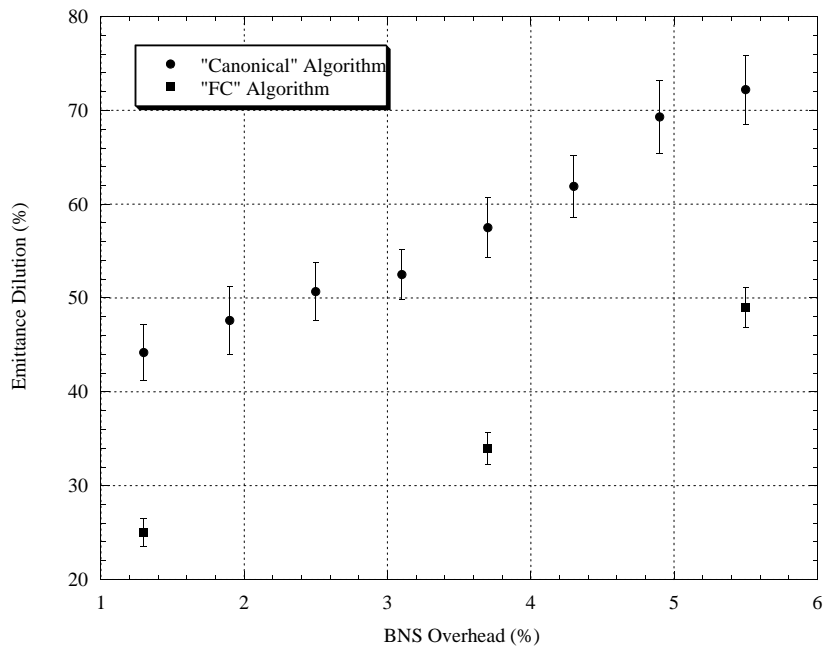

Figure 3: Emittance dilution as a function of energy overhead for "canonical" and "french curve" algorithms.

\section{DIFFUSIVE GROUND MOTION}

In recent years, Shiltsev [6] has offered evidence that accelerator alignment degrades according to a diffusive process. The so-called "ATL Law" states that components which are $a b$ initio perfectly aligned will be misaligned by an RMS distance $\sigma$ which is related to the distance between the components $L$ and the elapsed time $T$ by:

$$
\sigma^{2}=A \cdot T \cdot L
$$

The coefficient $A$ is a complex function of site geology, cultural noise, and construction techniques. Furthermore $A$ is not precisely constant in time, but is subject to change over the course of many years. However on the scale of seconds, days, or months, Equation 1 may represent a lower bound on achievable alignment performance.

In order to simulate ATL misalignments in the context of accelerator steering it is necessary to assume a value for $A$ and a time $T$ over which alignment occurs. For this study we assume that the NLC will have a value of $A$ of $5 \times 10^{-7} \mu^{2} /$ meter/second, which is low but not unachievable. We assume that the initial steering of the accelerator from a coarse state of alignment (50 $\mu$ RMS misalignments) to convergence requires 1 minute per operation of quad or girder alignment (thus approximately 3 hours for the full linac), while subsequent steering operations require only 1 iteration per segment and only 30 seconds per operation. We assume that steering is performed constantly.

Figure 4 shows the performance of the "french curve" algorithm when ATL misalignments occur during steering.
Pass 1 in Figure 4 is the 3 hour, multi-iteration pass: the emittance dilution is increased from $34 \%$ to $65 \%$ by ATL misalignments. The subsequent, fast passes achieve an equilibrium emittance dilution of $50 \%$.

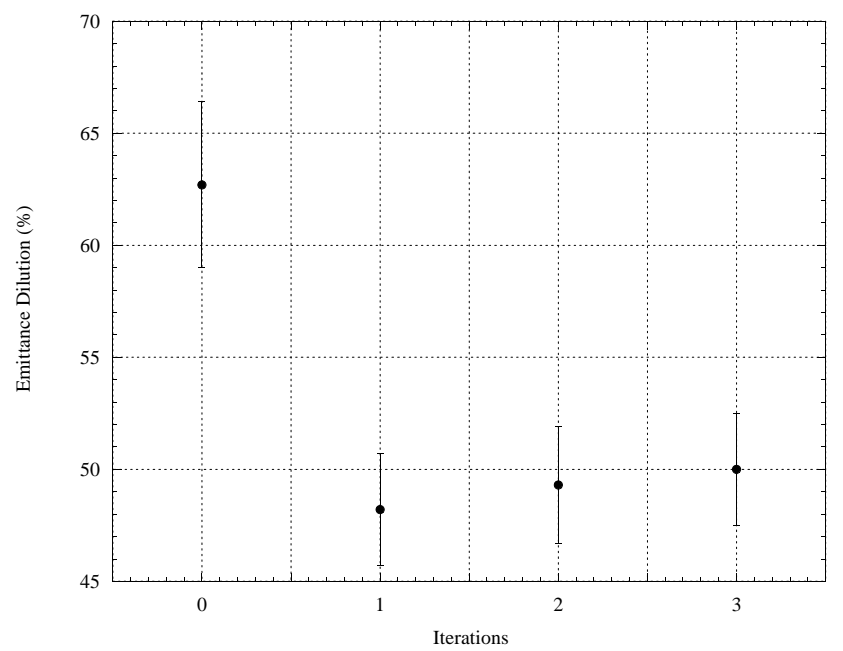

Figure 4: Performance of "french curve" algorithm with diffusive ground motion included.

\section{CONCLUSIONS}

We have evaluated several algorithms for steering the NLC main linac to reduce emittance dilution due to short-range wakefields and dispersion. We find that a relatively robust algorithm exists which produces acceptably small emittance dilution. Further studies of the algorithm are required. These include multibunch effects, improved modelling of the structure BPMs, interaction with steering feedbacks, and additional dilutions from other sources.

\section{ACKNOWLEDGEMENTS}

The authors wish to acknowledge the contributions of R. Assmann, T. Raubenheimer, and G. Stupakov.

\section{REFERENCES}

[1] NLC ZDR Design Group, "Zeroth-Order Design Report for the Next Linear Collider," SLAC Report 474 (1996).

[2] R.M. Jones et al, "Advanced Damped Detuned Structure (DDS) Development at SLAC." Proc. PAC 97 (1997).

[3] B. Autin and Y. Marti, "Closed Orbit Correction of A.G. Machines Using a Small Number of Magnets," CERN ISRMA/73-17 (1973).

[4] R. Assmann et al, "LIAR: A New Program for the Modeling and Simulation of Linear Accelerators with High Gradients and Small Emittances," Proc. LINAC 96, 464 (1996).

[5] V.E. Balakin, A.V. Novokhatsky, V.P. Smirnov, "VLEPP: Transverse Beam Dynamics," Proc. HEACC 83, 119 (1983).

[6] V. Shiltsev, "Space-Time Ground Diffusion: The ATL Law for Accelerators," Proc. IWAA 95, 352 (1996). 